\title{
Sound generation by a vortex ring collision
}

\author{
S. K. Tang ${ }^{\text {a) }}$ and N. W. M. Ko \\ Department of Mechanical Engineering, The University of Hong Kong, Hong Kong
}

(Received 19 April 1994; revised 8 November 1994; accepted 18 June 1995)

\begin{abstract}
A collision between two vortex rings and its sound generation is studied numerically using the contour dynamics method. Results suggest that the radial acceleration and the rate of change of the axial acceleration of the vortex rings are the more important dynamic parameters for sound generation during the interaction. The former is important at or near the pass-through instant when the vortex rings are coplanar and are of unequal strength. The latter, being important in the sound generation during a head-on collision before the vortex ring cores are very close to each other, especially when the rings are thin, is also important in sound generation by an unequal strength vortex ring collision after the pass through when the vortex cores are separated by a distance so that the mutual induction strength does not result in significant change of the impulse of the stronger ring. (C) 1995 Acoustical Society of America.
\end{abstract}

PACS numbers: 43.28.Ra, 43.28.Py

\section{INTRODUCTION}

Powell ${ }^{1}$ has shown that the source of aerodynamic sound in an unbounded low Mach number flow is related to the dynamics of flow vorticity. With the introduction of the coherent structure concept, Davis ${ }^{2}$ concluded that coherent structures, which usually bear vorticity, are the dominant structures in turbulent shear flow. In axisymmetric shear layers, they exist in the form of vortex rings. Pairing between these vortex rings and their eventual breakdown is believed to be the chief source of sound in a single circular jet. ${ }^{3,4}$ However, the actual motion of the vortical structures that generate sound is not specifically known. Laufer ${ }^{5}$ conjectured that sound was generated during pairing under high accelerating motion of the vortex rings. Recently, Tang and $\mathrm{Ko}^{6}$ showed both theoretically and experimentally the close relationship between the accelerating motion of vortical structures and the sound generation in a single circular jet.

The above-mentioned studies are relevant to the study of sound generation by a single circular jet in which the interacting vortical structures possess circulation of the same rotational direction (vortex pairing). However, it is shown experimentally by Tang and $\mathrm{Ko}^{7}$ that interaction between vortex rings with circulation of opposite sign occurs within coaxial jets with an "inverted velocity profile." 8 This is also the only type of vortex interaction recovered by a conditional sampling technique with the near-field pressure fluctuations as the triggering signals, ${ }^{9}$ showing that this interaction plays an important role in the generation of coaxial jet noise. As Tanna ${ }^{8}$ observed that the coaxial jet with an "inverted velocity profile" is less noisy than the equivalent single jet of the same thrust, the study of the aeroacoustics of the opposite sign vortex ring interaction is important not only in enhancing the basic understanding of vortex sound but may also provide clues for the control of coaxial jet noise. However, in the present stage, it is more desirable to study the simple form of vortex ring interaction as the coherent structure in-

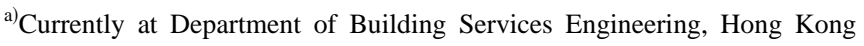
Polytechnic, Hong Kong. teraction in the coaxial jet is still too complicated to model.

Interaction of vortex rings with circulation of opposite direction of rotation moving toward each other, which is called "collision," 10 has been investigated by a number of researchers. However, few of them attempt to study the sound generation mechanism of the interaction. Oshima ${ }^{11}$ and Riley ${ }^{12}$ only studied the dynamics of the vortex rings. Kambe and Minota ${ }^{13}$ attempted to explain the role of viscosity in the generation of sound by the collision process while Shariff et al. ${ }^{14}$ showed that the inviscid model can reveal the essential features of the radiated sound time signals before the cores of the vortex rings are very close to each other. However, the actual sound generation mechanism is not yet understood.

In the present study, the contour dynamics method is employed to study the dynamics of the interacting vortex rings during their collision. The corresponding far-field sound-pressure fluctuations are calculated using the formula of Möhring. ${ }^{15}$ An attempt will also be made to relate the vortex ring motions with sound generation. These motions are more amenable to experimental investigation and it is hoped that the results can provide support for future theoretical and experimental studies.

\section{THEORETICAL CONSIDERATION AND NUMERICAL METHODS}

The flow considered in the present investigation is inviscid, axisymmetric, and free of swirl. Based on the contour dynamics method ${ }^{16,17}$ (Appendix), the velocity field induced by a vortex ring can be calculated once its core boundary $\mathbf{b}$ is known. The axial induced velocity $u$ and radial induced velocity $v$ at a point in the flow field with radial distance $\sigma_{p}$ are given, respectively, by

$$
\begin{aligned}
& u=\frac{1}{4 \pi} \int_{\mathbf{b}} \Omega \omega \hat{j} \cdot d \mathbf{b}, \\
& v=\frac{1}{\sigma_{p}} \int_{\mathbf{b}} \psi \omega \hat{j} \cdot d \mathbf{b},
\end{aligned}
$$


where $\Omega$ and $\psi$ are, respectively, the solid angle and stream function, ${ }^{17} \omega$ the vorticity, and $\hat{j}$ a unit tangent on the core boundary. The dynamic condition of the vorticity transport equation suggests that

$$
\omega=\lambda \sigma,
$$

where $\lambda$ is the vorticity constant ${ }^{18}$ and $\sigma$ the radial distance at a point within the vortex ring core. Here, $\lambda$ can be regarded as the radial vorticity gradient within the vortex core. If the core size is fixed, the larger the $\lambda$, the higher the circulation of the vortex ring. Thus this vorticity constant defines the strength of the vortex ring. The motion of the vortex ring core can then be solved using the fourth-order Runge-Kutta method once the core boundary $\mathscr{C}$ is defined at the instant when interaction commences. The numerical technique used in the calculation of $u$ and $v$ is the same as that employed by Pozrikidis ${ }^{17}$ and is not repeated here.

The far-field pressure fluctuations $p$ produced by vortex ring collision are also calculated by using contour integration. For a single vortex ring system, it can be inferred from Möhring's formula ${ }^{15}$ that

$$
p \propto \lambda \frac{\partial^{3}}{\partial t^{3}} \int_{\mathbf{b}} \sigma^{3} z^{2} \hat{j} \cdot d \mathbf{b},
$$

where $\sigma$ and $z$ are the radial and axial distance of a point on the core boundary. The integral in Eq. (3) is denoted by $S$ for easy reference and

$$
S=\int_{\mathbf{b}} \sigma^{3} z^{2} \hat{j} \cdot d \mathbf{b}
$$

The vortex impulse $I$ and the circulation $\Gamma$ can also be found using contour integrals as shown in Pozrikidis. ${ }^{17}$

In order to relate the physical motion of the vortex rings with sound generation, it is necessary to define the positions of the vortex rings during the interaction. The definition of vorticity centroid $\left(z_{c}, \sigma_{c}\right)$ in the present investigation follows that of Lamb: ${ }^{19}$

$$
\begin{aligned}
& \sigma_{c}^{2}=\int_{A} \omega \sigma^{2} d A / \int_{A} \omega d A=\frac{I}{\Gamma}, \\
& z_{c}=\int_{A} \omega \sigma^{2} z d A / \int_{A} \omega \sigma^{2} d A=\frac{S}{I} .
\end{aligned}
$$

It is noted that $S=I z_{c}$ and therefore

$$
\frac{\partial^{3} S}{\partial t^{3}}=I J_{z}+3 \varkappa_{z} \frac{\partial I}{\partial t}+3 V_{z} \frac{\partial^{2} I}{\partial t^{2}}+z_{c} \frac{\partial^{3} I}{\partial t^{3}},
$$

where the axial velocity $V_{z}=\partial z_{c} / \partial t$, axial acceleration $x_{z}=\partial^{2} z_{c} / \partial t^{2}$, and the axial jerk $J_{z}=\partial^{3} z_{c} / \partial t^{3}$. Equation (6) suggests the close relationship between the far-field pressure flucutations, vortex impulses, and axial motion of the vorticity centroids. The total sound radiated is the sum of $\partial^{3} S / \partial t^{3}$ of the two interacting vortex rings. Thus

$$
p \propto \sum I J_{z}+3 \sum \varkappa_{z} \frac{\partial I}{\partial t}+3 \sum V_{z} \frac{\partial^{2} I}{\partial t^{2}}+\sum z_{c} \frac{\partial^{3} I}{\partial t^{3}} .
$$

The terms on the right-hand side of Eq. (6) have physical meanings. The first term, $I J_{z}$, represents radiation due to a change in the axial acceleration of the vortex ring of impulse $I$. As the rate of change of $I$ represents a force acting on the vortex ring, the second term, $\varkappa_{z} \partial I / \partial t$, and the third term, $V_{z} \partial^{2} I / \partial t^{2}$, denote the radiation resulting from the change in fluid power due to axial accelerating motion and the change in vortex impulse, respectively. In the case of two vortex ring interaction, these two terms together represent acoustic radiation from an unbalance in the change of fluid power of the vortex rings due to internal forces. As there is no external force acting on the system, these forces are due to mutual induction between the vortex rings. The last term, $z_{c} \partial^{3} I / \partial t^{3}$, is the part of the radiation associated with the relative axial position of the vortex rings in the presence of nonlinearly time varying forces.

In the present investigation, the contour integrals are solved by the fourth-order Gauss-Legendre quadrature method. ${ }^{17}$ The time differentiation is done by the method of central difference. Also, the initial shape of the vortex ring cores follows the solutions of Norburg ${ }^{19}$ which are characterized by the effective core size $\alpha$. Here, $\alpha$ is defined by the equation

$$
\alpha^{2}=\text { initial area of the vortex ring core } / \pi R^{2} \text {. }
$$

The lengths involved in the computation are normalized by the initial radius of the vorticity centroid $R$. The time $t$ is normalized by $\omega_{0}^{-1}$, where $\omega_{0}=\lambda R$ and $\lambda$ is associated with the vortex ring of weaker circulation. All other quantities presented in this paper are dimensionless unless otherwise specified. The velocity $V_{z}$, the acceleration $\varkappa_{z}$ and $\varkappa_{\sigma}$, the axial jerk $J_{z}$, and the vortex impulses $I$ are normalized, respectively, by $R \omega_{0}, R \omega_{0}^{2}, R \omega_{0}^{3}$, and $R^{4} \omega_{0}$. The proportionality constant and the directivity factor in the far-field pressure equation [Eq. (3)] are not related to the vortex ring dynamics and are neglected in the present investigation. The far-field pressure fluctuations $p$ are normalized by $R^{7} \omega_{0}^{2}$. Also, anticlockwise circulation is treated as positive while the clockwise one as negative.

The computations were done on the UNIX Sun system and DECstation ULTRIX 4.2 system in The University of Hong Kong.

\section{RESULTS AND DISCUSSIONS}

Figure 1 shows a schematic sketch of the collision system. The initial axial separation of the vorticity centroids and the initial radius of the vortex ring are denoted by $C$ and $R$, respectively. Here, $\mathbf{b}_{L}$ and $\mathbf{b}_{R}$ represent the core boundaries of the leftward and rightward propagating rings, respectively. The rightward propagating vortex ring possesses anticlockwise (positive) circulation while the other possesses clockwise circulation (negative). During collision, initially, the vortex rings decelerate axially, and their radii increase as they move toward each other. The sizes of their cores decrease due to the conservation of vortex volume. It will be shown in the following two sections that the vortex ring dynamics and the sound generation mechanism depend significantly on the circulation ratio of the two colliding vortex rings.

The "permanent union" of vortex rings, as discussed by Hicks, ${ }^{20}$ is not observed in the present study. However, since 


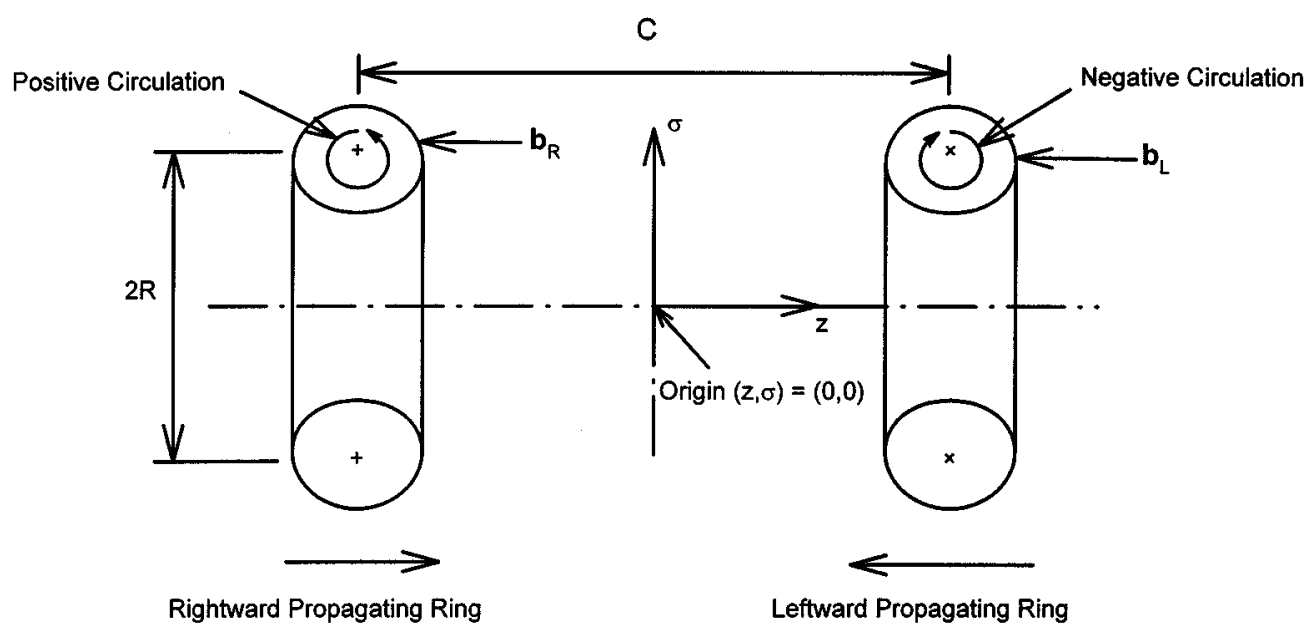

FIG. 1. Schematic sketch of collision system.

the vortex rings under such conditions are propagating with the same and constant speed and are coplanar, the term $\partial^{3} S / \partial t^{3}$ in Eq. (6) vanishes. Such interaction is silent or at least the amplitude of radiation is insignificant.

\section{A. Head-on collision}

Head-on collision occurs when the colliding vortex rings are identical except they have opposite circulation directions. Figure 2 shows the dynamics of the rightward propagating vortex ring core calculated using the contour dynamics method (see the Appendix) for $\alpha=0.5$ and initial core separation $C / R=4$ at the normalized time of flight $\omega_{0} t=0,12$, and 21 . The dynamics of the leftward propagating vortex ring are the mirror images about the plane $z / R=0$ of those shown in Fig. 2 and thus are not presented. The solid line represents the core boundary and the symbol + denotes vorticity centroid. In the theory of contour dynamics, the radii of the vortex rings will increase continuously. However, computation was stopped at $\omega_{0} t=25$ when the core boundaries of the vortex rings are very close to each other as the vortices split into smaller rings ${ }^{11}$ and such a phenomenon cannot be

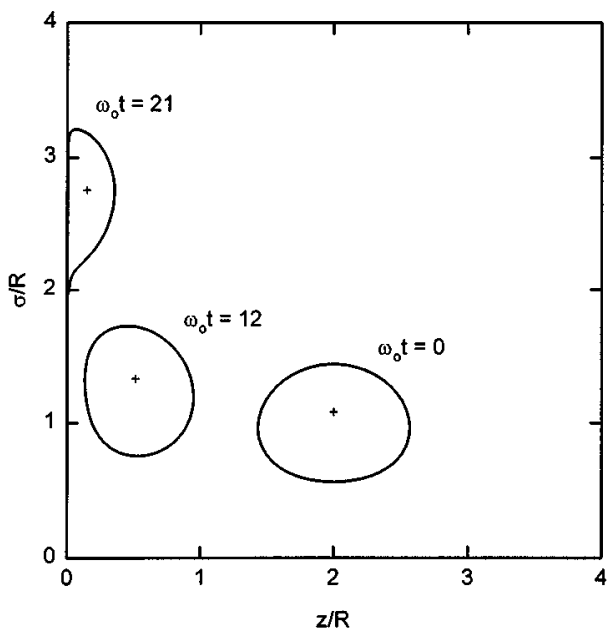

FIG. 2. Vortex ring core evolution under equal strength vortex collision. $\alpha=0.5, \gamma=1, C / R=4, \omega_{0} t=0,12,21 . \stackrel{\longrightarrow}{\longrightarrow}$ : Vortex core boundary; + vorticity centroid.
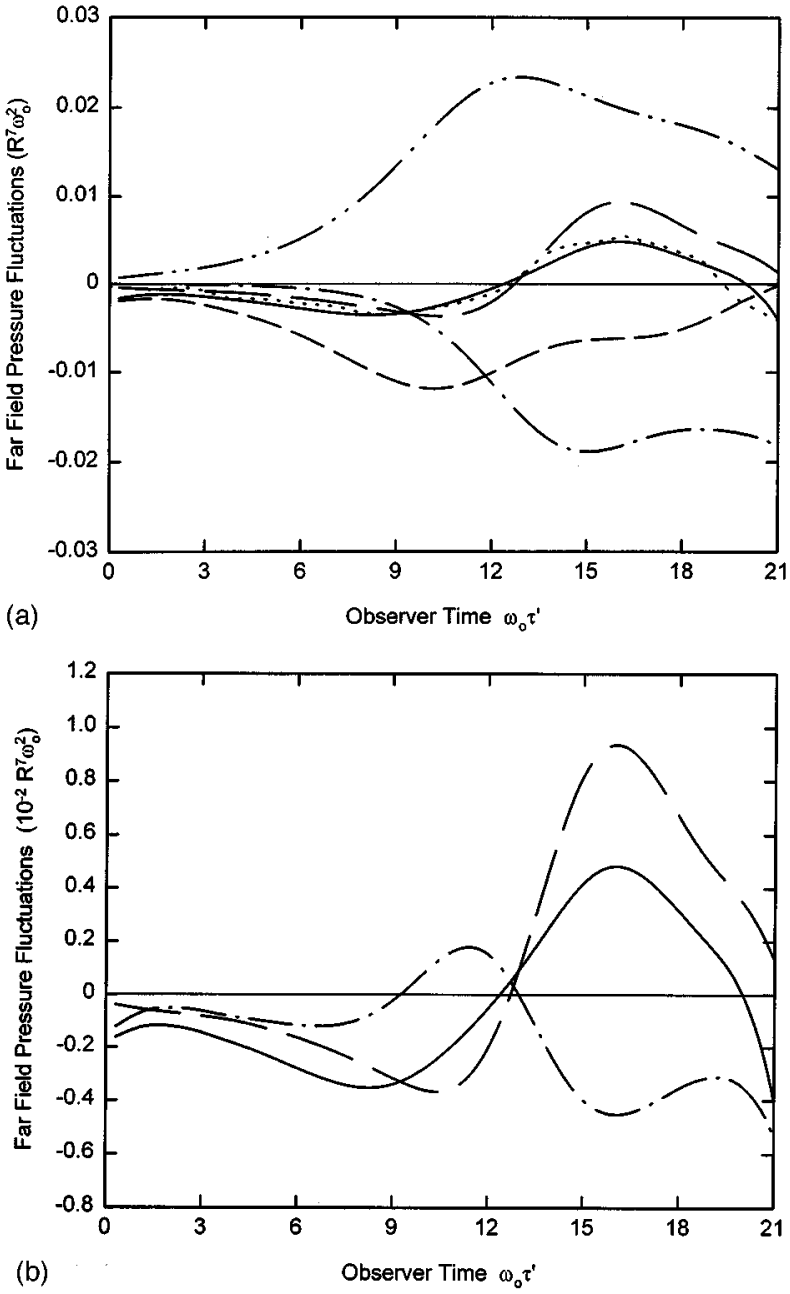

FIG. 3. (a) Far-field pressure fluctuations against observer time $\tau^{\prime}$. Total sound-pressure fluctuations; --: sound related to axial jerk, $\Sigma I J_{z}$; $-\cdot-$ : sound related to rate of change of fluid power due to axial acceleration, $3 \sum \varkappa_{z} \partial I / \partial t ;-\cdots-$ : sound related to rate of change of fluid power due to rate of change of mutual induction strength, $3 \Sigma V_{z} \partial^{2} I / \partial t^{2}$; ----: sound radiated by nonlinearity of mutual induction strength time variations, $\Sigma z_{c} \partial^{3} I / \partial t^{3}$; …..: Kambe and Minota (see Ref. 13). (b) Contribution of $\Sigma I J_{z}$ in the sound generation. ——: Far-field pressure fluctuations; ---: sound associated with axial jerk, $\Sigma I J_{z} ;-\cdot-$ : far-field pressure fluctuations excluding the contribution of $\Sigma I J_{z}, \alpha=0.5, \gamma=1, C / R=4$. 
accurately reproduced by the method of contour dynamics. Similar vorticity dynamics, as in Fig. 2, can be found in Shariff et al., ${ }^{14}$ though at different values of $\alpha$. Both Shariff and Leonard ${ }^{10}$ and Shariff et al. ${ }^{14}$ had not studied the details of the sound generation process. Thus this paper is an attempt to deal with this issue.

The corresponding far-field pressure fluctuations and the contributions of the four terms $\sum I J_{z}, 3 \sum \varkappa_{z} \partial I / \partial t$, $3 \sum V_{z} \partial^{2} I / \partial t^{2}$, and $\sum z_{c} \partial^{3} I / \partial t^{3}$ are plotted against the observer time $\tau$ in Fig. 3(a). The observer time $\tau$ is defined as

$$
\tau=t+r / a_{0},
$$

where $t$ is the emission time, $r$ is the far-field distance, and $a_{0}$ the ambient speed of sound. For easy presentation, the abscissa in Fig. 3(a) is chosen to be $\omega_{0} \tau^{\prime}$ where $\tau^{\prime}=\tau-r / a_{0}$. Although $\tau^{\prime}=t$, it is used here to distinguish between emission and observer times. The experimental results of Kambe and Minota ${ }^{13}$ are also included for comparison. It can be observed that the present calculated total farfield pressure fluctuations agree well with those of Kambe and Minota. ${ }^{13}$ The observed small discrepancy is probably due to the unknown core size and initial separation of the vortex rings in the experimental results of Kambe and Minota. ${ }^{13}$ This suggests that the inviscid model can predict the noise radiated by vortex ring collision before the vortex core boundaries are very close to each other. Viscosity, therefore, becomes important only after this instant. However, the present calculated results successfully reveal the further decrease of far-field pressure at $\omega_{0} \tau^{\prime}>18$ which is not observed in the calculation of Shariff et al. ${ }^{14}$

It is also noted that the variation of the total far-field pressure fluctuations follows closely that of the first radiation term $\sum I J_{z}$. The other three terms, $3 \sum \varkappa_{z} \partial I / \partial t$, $3 \sum V_{z} \partial^{2} I / \partial t^{2}$, and $\Sigma z_{c} \partial^{3} I / \partial t^{3}$, though having higher amplitudes, tend to counteract each other so that in general they totally contribute about $50 \%$ of the sound radiated [Fig. 3(b)]. It seems that the axial jerks $J_{z}$ and impulses $I$ of the vortex rings are the relatively more important parameters in the sound generation process. It will be shown later that they are also important in the sound generation of thin vortex ring collision before the two vorticity centroids are very close to each other.

Figure 4 illustrates the time variations of axial jerk $J_{z}$ and impulse $I$ of the rightward propagating vortex ring. Those of the other vortex ring differ only in sign and thus are not presented. The amplitude of the impulse increases monotonically as the vortex rings approach each other, while the total impulse $\Sigma I$ of the system remains essentially zero. Thus the fluctuations in the first radiation term $\Sigma I J_{z}$ are mainly due to the axial jerk $J_{z}$.

The far-field pressure fluctuations of thin vortex ring collisions for $\alpha=0.1$ are shown in Fig. 5. Computation was stopped at $\omega_{0} t=270$. It is clear that the far-field pressure time variation $p$ at $\omega_{0} \tau^{\prime} \leqslant 210$ collapses with that of $\sum I J_{z}$, showing that the total contribution of the other three terms is much less significant during this period. Though there is an observable difference between $p$ and $\sum I J_{z}$ at $\omega_{0} \tau^{\prime}>210$, the latter term still contributes more than $50 \%$ of the radiated sound. The overall correlation coefficient between $p$ and the

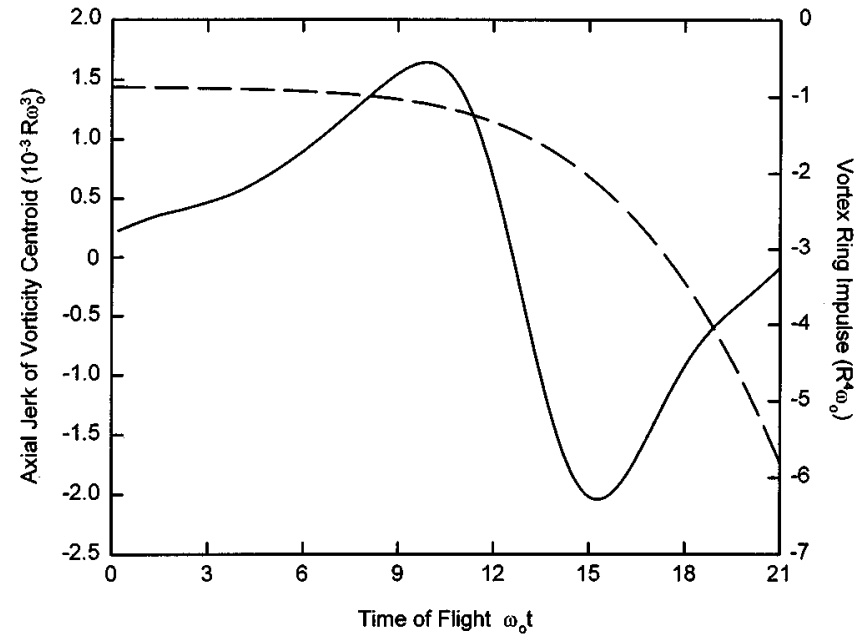

FIG. 4. Time variations of axial jerk and vortex ring impulse. $\alpha=0.5, \gamma=1$, $C / R=4$. - Axial jerk of vortex ring, $J_{z} ;---$ : vortex ring impulse $I$.

term $\sum I J_{z}$ is around 0.9 , showing the importance of the axial jerk and vortex impulse in the generation of sound. Wavy oscillations, which have been found by Shariff et al. ${ }^{14}$ in the study of vortex ring pairing sound, appear in the farfield pressure fluctuations $p$ and $\sum I J_{2}$ for $\alpha=0.1$ (Fig. 5). The oscillations in $\Sigma I J_{z}$ observed in the present investigation are due to the axial jerks of the vortex rings (not shown here). Figures 3 and 5 suggest that the importance of axial jerk in sound generation decreases as the core sizes of vortex rings increase. However, further investigation is needed to find the physical explanation for the phenomenon.

Since the interacting coherent structures within the coaxial jet are usually of different strengths, ${ }^{7}$ the collision of unequal strength vortex rings is more important in its noise generation.

\section{B. Collision between vortex rings of unequal circulations}

When the magnitudes of circulation of the two vortex rings are different, their cores eventually move away from

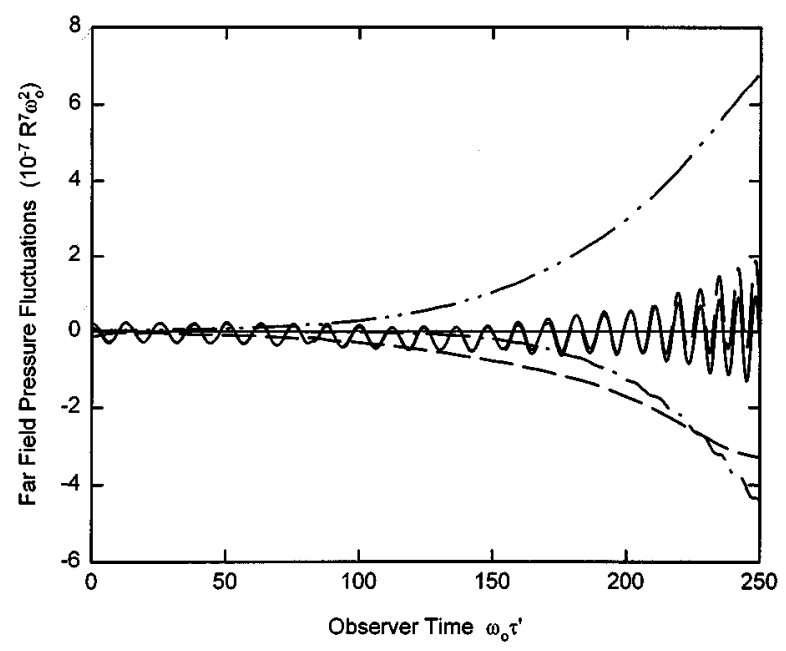

FIG. 5. Time variations of far-field pressure fluctuations for thin vortex ring collision. $\alpha=0.1, \gamma=1, C / R=4$. Legends: same as those in Fig. 3(a). 

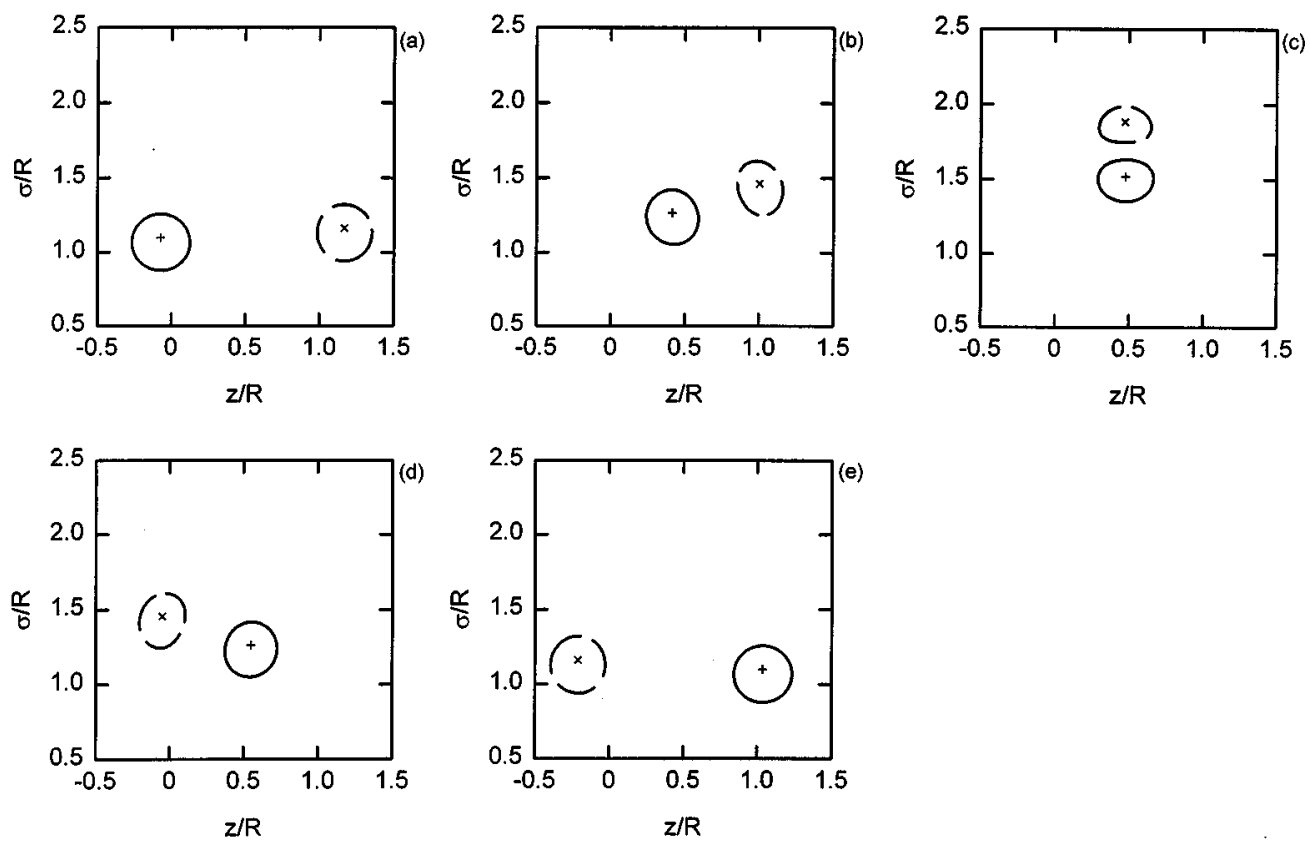

FIG. 6. Vortex ring core evolution for unequal strength vortex ring collision. $\alpha=0.2, \gamma=2, C / R=4$. (a) $\omega_{0} t=30$; (b) $\omega_{0} t=40$; (c) $\omega_{0} t=50$; (d) $\omega_{0} t=60$; (e) $\omega_{0} t=70$. —: Strong ring core boundary; --: weak ring core boundary; +: strong ring vorticity centroid; $\times$ : weak ring vorticity centroid.

each other, because of the difference in their directions of propagation. However, the shapes of the cores depend significantly on the circulation ratio and the effective core sizes $\alpha$ of the vortex rings. The radii of the vortex rings attain their respective maxima at or near the instant when their vorticity centroids are coplanar (Fig. 6). This instant is referred to as the pass-through instant in the present study. The radial velocities of the vorticity centroids vanish or become very small around this instant. The circulation ratio is defined as $\gamma=\left|\Gamma_{R} / \Gamma_{L}\right|$, where the suffices $L$ and $R$ denote quantities associated with the leftward and rightward propagating vortex rings, respectively. The vortex ring having a smaller circulation magnitude is named the weak ring while the other is called the strong ring.

When the core size is small, the vortex rings finally separate from each other without significant core deformation. Figure 6 is a typical example of such an interaction where the vortex core dynamics are shown for $\alpha=0.2, \gamma=2$, and $C / R=4$. The time variations of the axial positions of the vorticity centroids are shown in Fig. 7 , in which the strong ring is found to move in a direction opposite to its original one at or near the pass-through instant $\omega_{0} t=50$. This shows that there are considerable changes in its velocity, acceleration, and jerk during this period of time which may result in high intensity sound radiation.

The corresponding time variations of the total far-field pressure fluctuations and the four terms on the right-hand side of Eq. (7) are shown in Fig. 8. The interaction gives rise to a sound pulse at $\omega_{0} \tau^{\prime}=50$, which corresponds to sound generation at the pass-through instant (Fig. 7). The amplitude of the sound pulse depends very much on the term $3 \Sigma V_{z} \partial^{2} I / \partial t^{2}$, which represents the net rate of change of fluid power due to a change in the mutual induction strength between the vortex rings. Figure 9(a) illustrates that the weak ring contributes more to the appearance of the sound pulse.
The counteraction between the contributions of the two vortex rings is due to the temporary change in propagation direction of the strong ring under the induced velocity field of the weak ring (Fig. 7). The term $V_{z} \partial^{2} I / \partial t^{2}$ is associated with the axial velocity $V_{z}$, radial velocity $V_{\sigma}$, and radial acceleration $\varkappa_{\sigma}$ as

$$
\frac{\partial^{2} I}{\partial t^{2}}=\frac{\partial^{2}}{\partial t^{2}} \Gamma \sigma_{c}^{2}=2 \Gamma\left(V_{\sigma}^{2}+\sigma_{c} \varkappa_{\sigma}\right),
$$

where the rate of change of $\Gamma$ is negligible. Since the radial velocity $V_{\sigma}$ vanishes or becomes sufficiently small at or near the pass-through instant $\omega_{0} t=50$, it does not contribute much to the sound pulse. The time variations of the axial velocities and radial accelerations of the vortex rings are

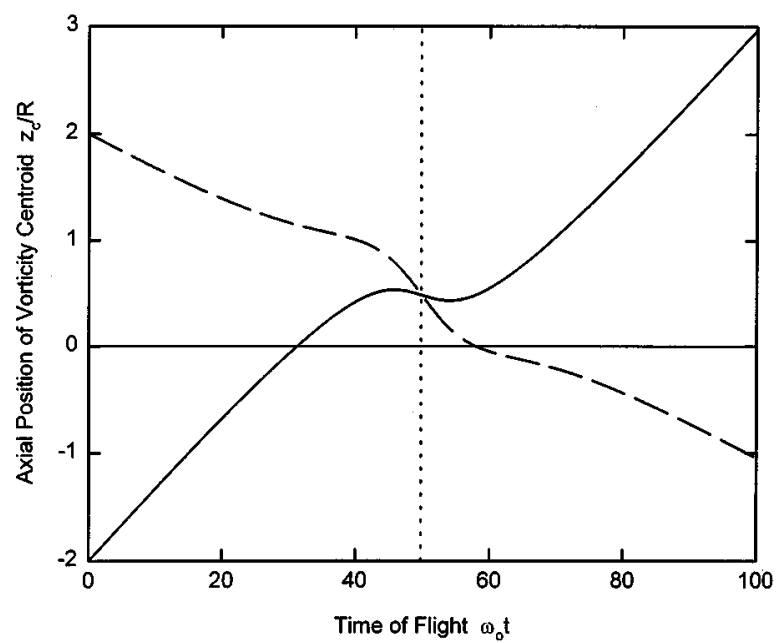

FIG. 7. Time variations of axial positions of vorticity centroids. $\alpha=0.2$, $\gamma=2, C / R=4$. — : Strong ring; ---: weak ring; $\cdots$ : pass-through instant. 


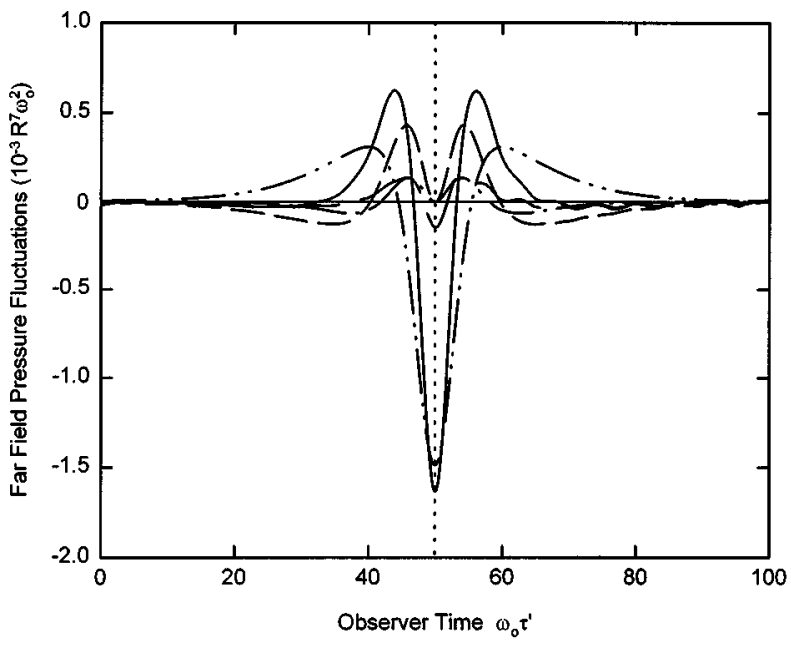

FIG. 8. Far-field pressure fluctuations against observer time $\tau^{\prime} . \alpha=0.2$, $\gamma=2, C / R=4$. $\cdots$ : pass-through instant; other legends: same as those in Fig. 3(a).

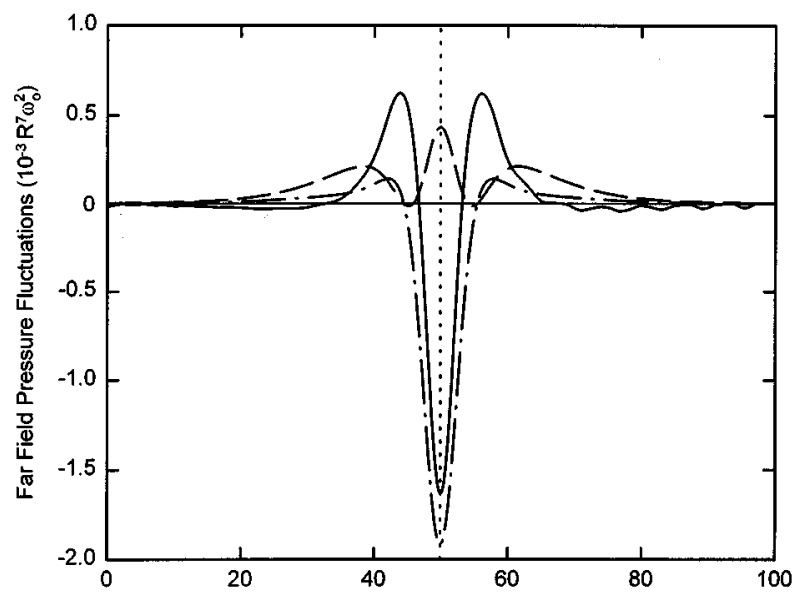

(a)

Observer Time $\omega_{0} \tau^{\prime}$

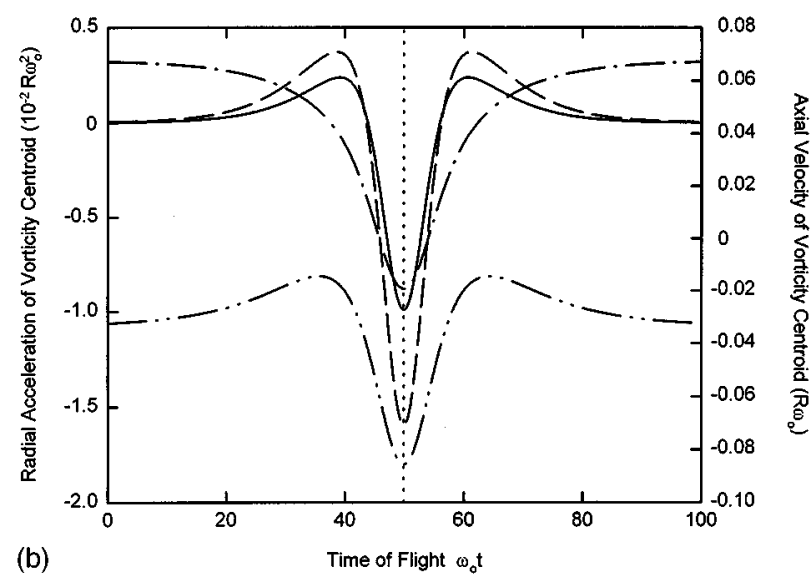

FIG. 9. (a) Acoustic contributions of unequal strength vortex rings. $\alpha=0.2$, $\gamma=2, C / R=4$. — Total sound pressure; -- : strong ring; - - - : weak ring; $\cdots$ : pass-through instant. (b) Time variations of axial velocity and radial acceleration. $\alpha=0.2, \gamma=2, C / R=4$. — Radial acceleration of strong ring; - -: radial acceleration of weak ring; - --: axial velocity of strong ring; $-\cdots-$ : axial velocity of weak ring; $\cdots \cdots$ : pass-through instant. shown in Fig. 9(b). It is noted that both the time variations of the radial acceleration and axial velocity, especially the former, agree with that of the far-field pressure fluctuations, suggesting that the vortex rings radiate high noise levels at the pass-through instant when their radial accelerations and axial velocities are the highest during the collision. Figures 8 and 9(b) also show that the positive and negative values of the radiation term $3 \Sigma V_{z} \partial^{2} I / \partial t^{2}$, which is associated with the axial velocity and radial acceleration of the weak ring, come mainly from the radial acceleration of the weak ring as its axial velocity is always negative so that the form and magnitude of the sound pulse are basically determined by the time variation of the radial acceleration. Therefore, the radial acceleration becomes relatively more important than other dynamic parameters in sound generation.

The wavy oscillations observed in Fig. 8 at $\omega_{0} \tau^{\prime}>68$ after the pass-through instant are due to the axial jerk of the strong ring [Fig. 10(a)]. Though the amplitudes of the jerks reach a maximum at the pass-through instant, the opposite signs of circulation of the colliding vortex rings result in small $\sum I J_{z}$, contributing little to the appearance of the sound pulse. Figure 10(b) gives the time variations of the far-field pressure fluctuations for $\gamma=4$ and $\alpha=0.2$. It is also an example showing that the amplitude and the relative importance of the wavy oscillations increase with $\gamma$. The collapse of $p$ and $\Sigma I J_{z}$ at $\omega_{0} \tau^{\prime}>35$ can also be observed in Fig. 8 at $\omega_{0} \tau^{\prime}>68$. In order to show this similarity, the portion of $\omega_{0} \tau^{\prime}>60$ in Fig. 8 is magnitude and presented in Fig. 10(c). Thus the collapse of $p$ and $\Sigma I J_{z}$ in Fig. 10(b) and (c) further suggests the importance of the axial jerk in sound generation as the vortex rings are moving away from each other. Other results, not shown here, of higher circulation ratio $\gamma(\leqslant 10)$ indicate that the amplitude of the sound pulse also depends mainly on the term $3 \Sigma V_{z} \partial^{2} I / \partial t^{2}$.

It is also noted in Fig. 10(b) that the collapse of $p$ and $\sum I J_{z}$ at $\omega_{0} \tau^{\prime}<18$ is very similar to the results of the equal strength thin vortex ring collision shown in Fig. $5(\gamma=1$, $\alpha=0.1$ ). The collapse of $p$ and $\sum I J_{z}$ in Fig. 10(b) is even better than that shown in Fig. 10(c), showing that the similarity of the time variation patterns of $p$ and $\Sigma I J_{z}$ found in the equal strength vortex ring collision case, especially for thin vortex ring interaction, is not a curiosity. However, further study is required to establish the physical meaning of their relationship.

A higher effective core size of $\alpha=0.4$ results in the deformation of the vortex ring cores during their collision and eventual separation (Fig. 11). Figure 11 shows the dynamics of the vortex ring cores and the core deformation of the weak ring for $\gamma=8$ and $C / R=4$. A thin wisp of vorticity from the weak ring wipes around the core of the strong ring as the separation between the vorticity centroids increases after the pass-through instant [Fig. 11(e)]. This is probably due to the strong induced velocity field of the strong ring and the small separation between the vortex ring core boundaries close to the pass-through instant $\omega_{0} t \simeq 4.7$ [Fig. 11(c)]. Besides experiencing serious core deformation, the vorticity centroid of the weak ring is nearly stationary after the strong ring has completely passed through it at $\omega_{0} t \simeq 6$ (Fig. 12). The axial components of its velocity, acceleration, and jerk then vanish 


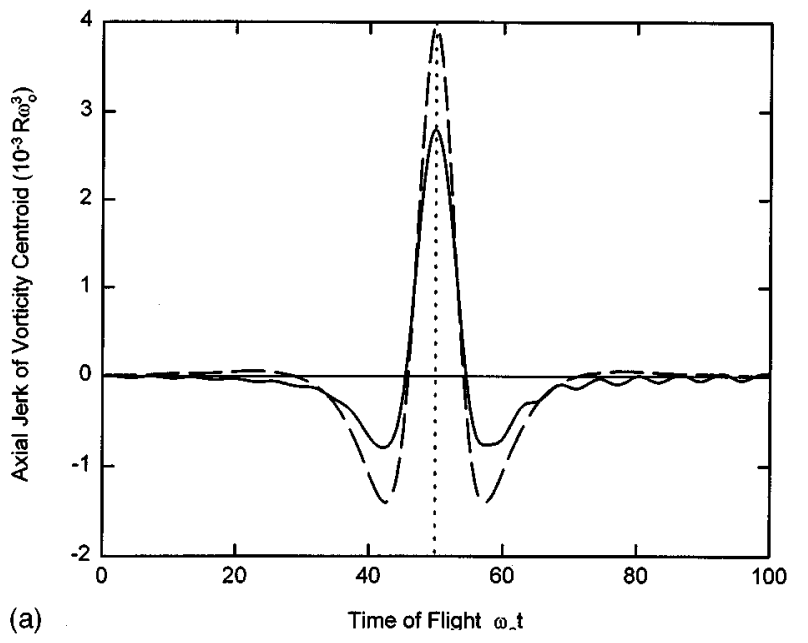

(a)

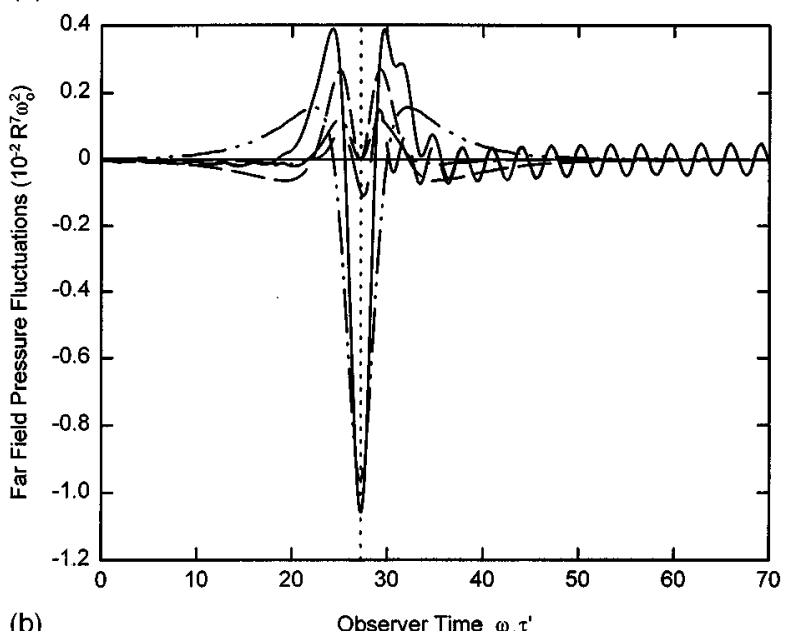

(b)

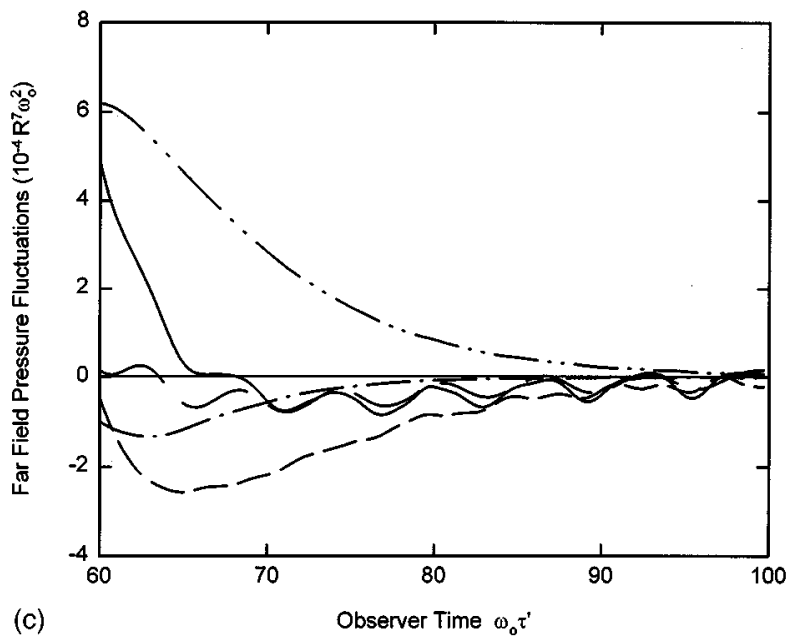

FIG. 10. (a) Time variations of axial jerk. $\alpha=0.2, \gamma=2, C / R=4$.

Strong ring; ---: weak ring; $\cdots$ : pass-through instant. (b) Far-field pressure fluctuations for $\gamma=4 . \alpha=0.2, \gamma=4, C / R=4 . \cdots$ : pass-through instant; other legends: same as those in Fig. 3(a). (c) Far-field pressure fluctuations for $\gamma=2$ at $\omega_{0} \tau^{\prime}>60 . \alpha=0.2, \gamma=2, C / R=4$. Legends: same as those in Fig. $3(\mathrm{a})$.

or become very small. As the changes in two vortex ring radii are small as flight time increases, the third time derivative of $S$, that is, $\partial^{3} S / \partial t^{3}$, associated with the weak ring becomes insignificant in sound generation at $\omega_{0} t \geqslant 6$. Thus the sound radiated depends mainly on the motion of the
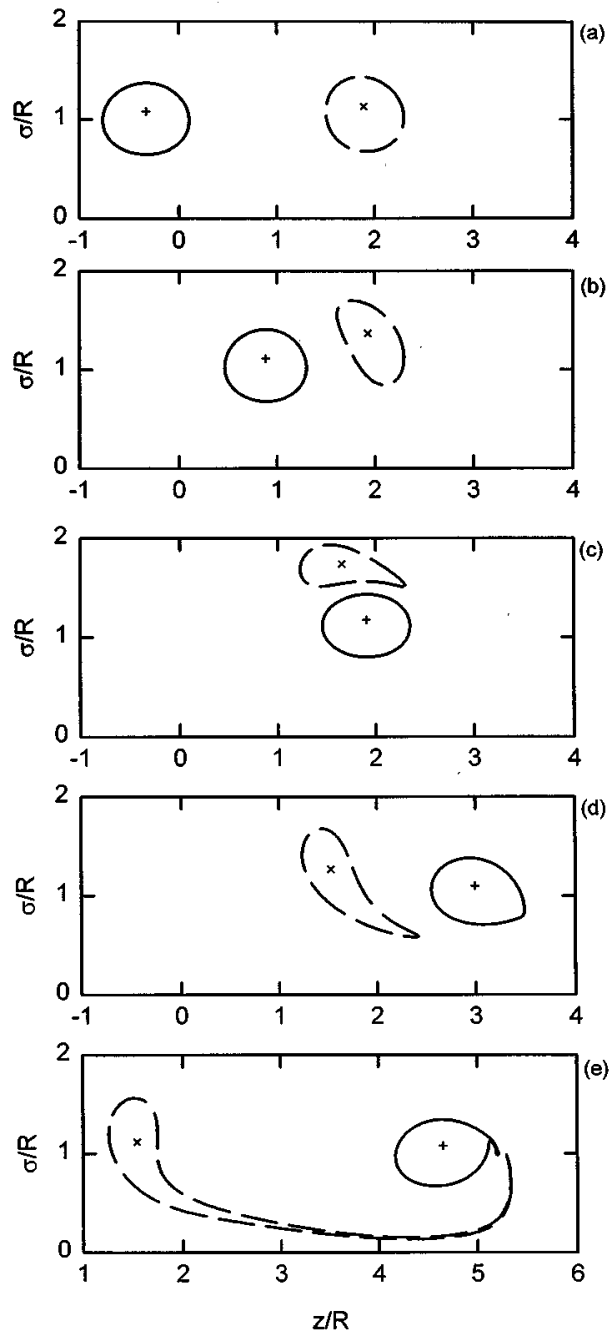

FIG. 11. Vortex ring core evolution for high circulation ratio. $\alpha=0.4, \gamma=8$, $C / R=4$. (a) $\omega_{0} t=2$; (b) $\omega_{0} t=3.5$; (c) $\omega_{0} t=5$; (d) $\omega_{0} t=6.5$; (e) $\omega_{0} t=8.5$. Other legends: same as those in Fig. 6 .

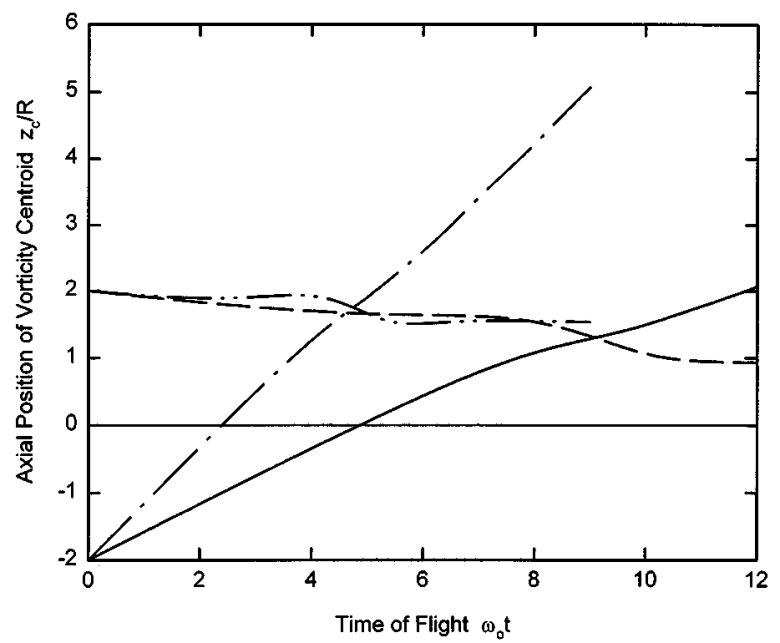

FIG. 12. Axial positions of vorticity centroids. $\alpha=0.4, C / R=4$.

Strong ring, $\gamma=4 ;--$ : weak ring, $\gamma=4 ;-\cdot-$ : strong ring, $\gamma=8 ;-\cdot--$ : weak ring, $\gamma=8$. 


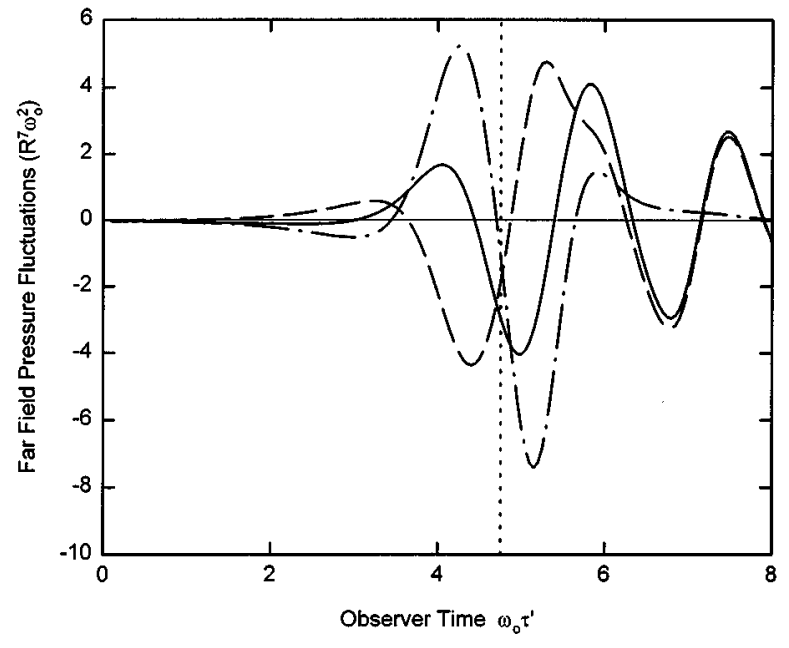

FIG. 13. Acoustic contributions of vortex rings for high circulation ratio. $\alpha=0.4, \gamma=8, C / R=4$. Legends: same as those in Fig. 9(a).

strong ring when the two vortex cores are separating from each other at increased flight time (at $\left.\omega_{0} \tau^{\prime}>6\right)$ (Fig. 13).

Figure 14 shows the corresponding far-field pressure fluctuations and the time variations of the four terms $\Sigma I J_{z}$, $3 \sum \varkappa_{z} \partial I / \partial t, 3 \sum V_{z} \partial^{2} I / \partial t^{2}$, and $\sum z_{c} \partial^{3} I / \partial t^{3}$. The time variation pattern of the far-field pressure fluctuations before the strong ring completely passes through the weak ring is similar to that observed for $\alpha=0.2$ (Fig. 8). The dip on the total far-field pressure fluctuations at $\omega_{0} t \simeq 4.9$, just after the pass-through instant $\omega_{0} t=4.7$, is mainly due to the term $3 \sum V_{z} \partial^{2} I / \partial t^{2}$, implying radial acceleration is again relatively more important in the sound generation close to the pass-through instant. At $\omega_{0} t \geqslant 6$, the term $\sum I J_{z}$ dominates the far-field pressure fluctuations. The observed large fluctuations and the amplitude in the far-field pressure during this period are due to the axial jerk of the strong ring, as there is only less than $5 \%$ change in its impulse at $\omega_{0} t>6$ (Fig. 15). When the mutual induction strength between the vortex rings is not high enough to cause significant change in the impulse of a particular vortex ring, the sound generated by this ring is primarily due to the unsteady core rotation or

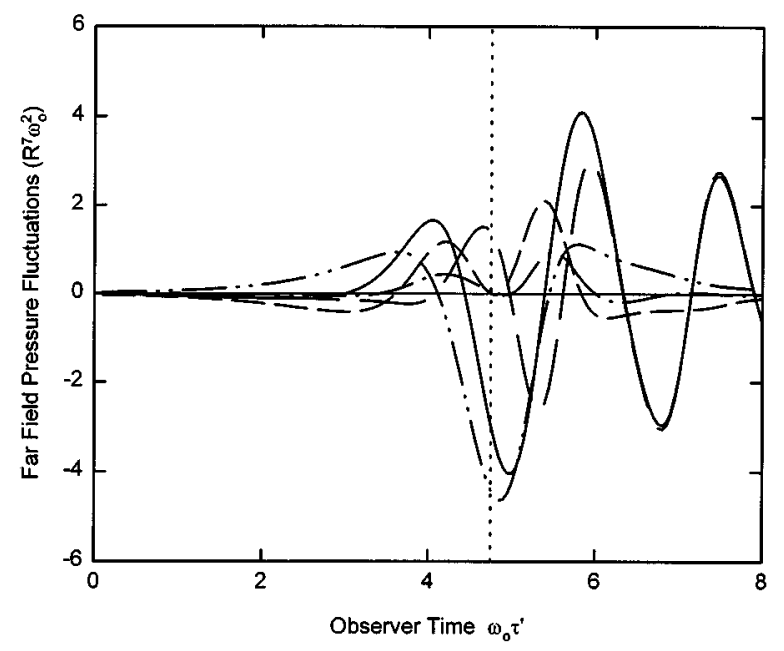

FIG. 14. Far-field pressure fluctuations against observer time $\tau^{\prime}$. $\alpha=0.4$, $\gamma=8, C / R=4$. Legends: same as those in Fig. 8 .

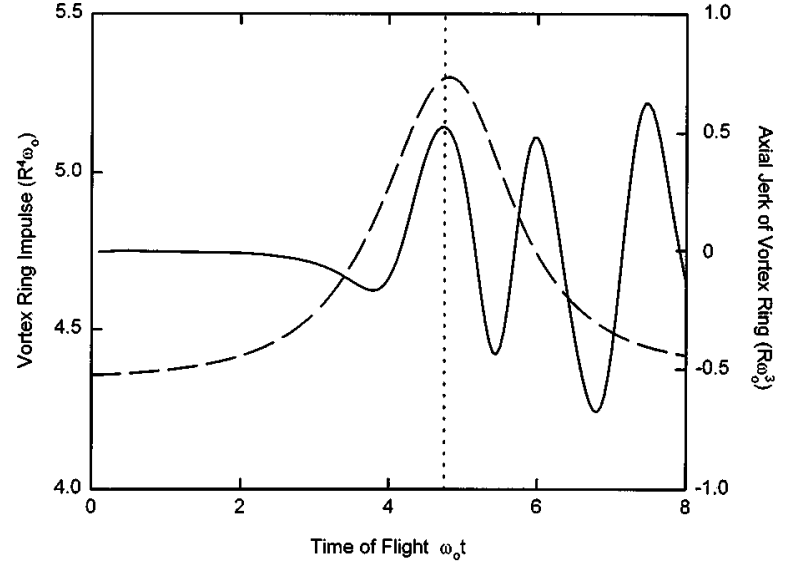

FIG. 15. Time variations of vortex impulse and axial jerk. $\alpha=0.4 ; \gamma=8$, $C / R=4$. . . : Pass-through instant; other legends: same as those in Fig. 4.

nutation. The only term that describes this effect is $I J_{z}$ so that there appears to be high correlation between this term and the far-field pressure fluctuations at $\omega_{0} \tau^{\prime}>6$ when the vortex cores are separated by more than one initial vortex ring radius.

A decrease in the circulation ratio $\gamma$ to 4 does not have much impact on the vortex ring dynamics, except that more serious deformation of the strong ring core occurs [Fig. 16(a)]. Again, the axial position of the weak ring vorticity changes very slowly with time after the pass-through instant $\omega_{0} t=8.2$ (Fig. 12). Its motion is then no longer important in
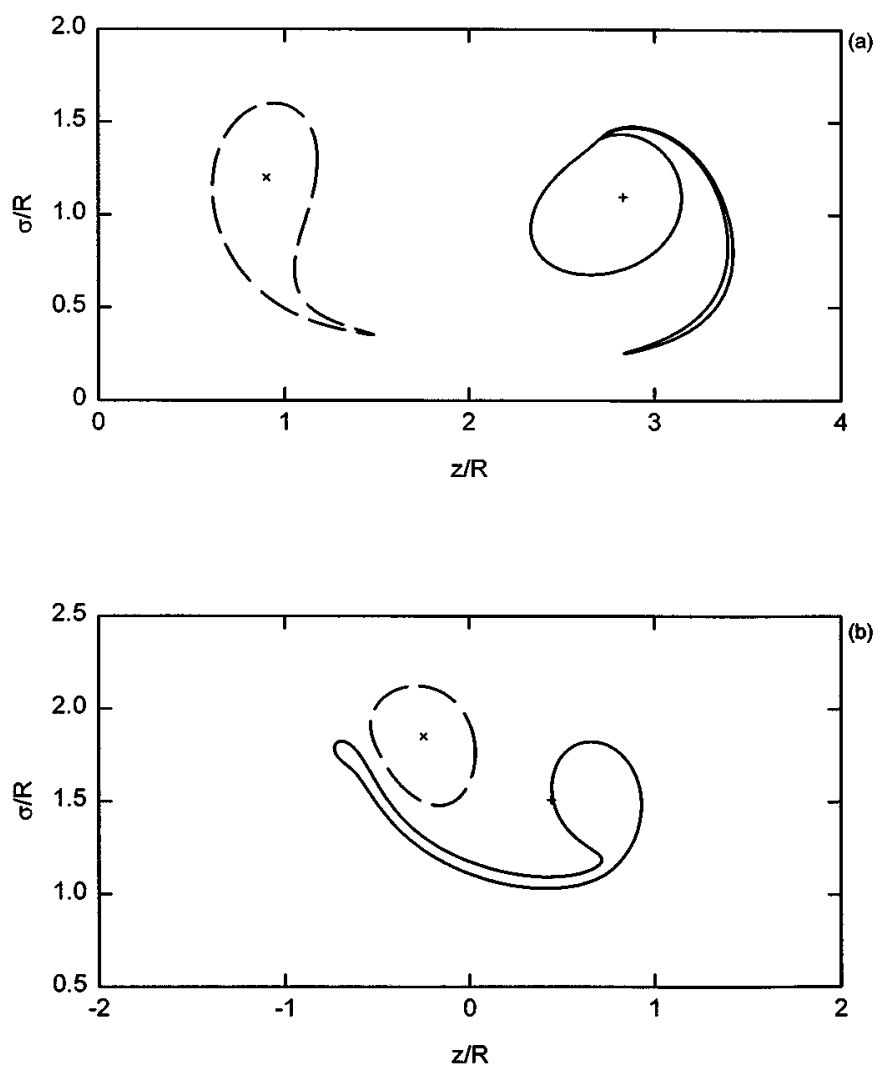

FIG. 16. Comparison of vortex ring cores evolution for $\gamma=2$ and 4. (a) $\alpha=0.4, \gamma=4, C / R=4, \omega_{0} t=14$; (b) $\alpha=0.4, \gamma=2, C / R=4, \omega_{0} t=22$. Legends: same as those in Fig. 6. 


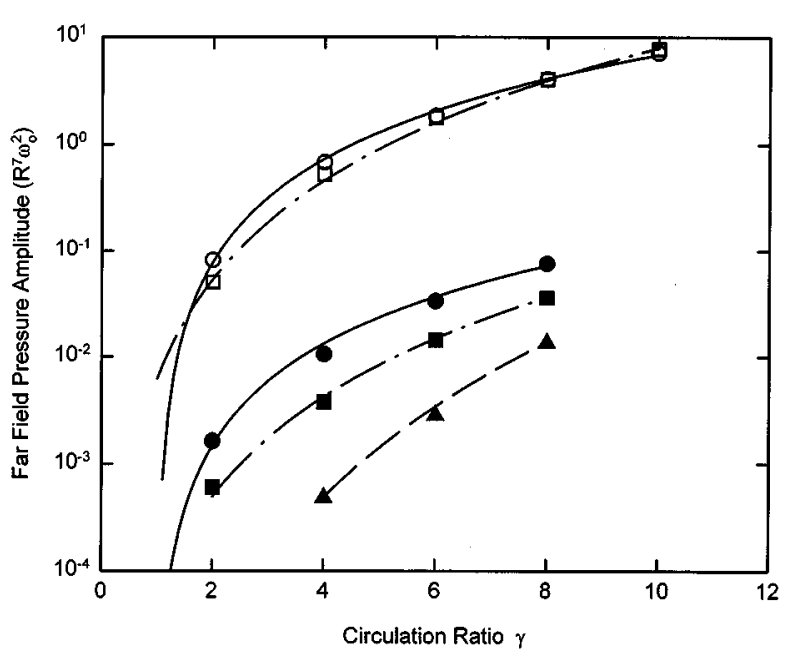

FIG. 17. Variations of far-field pressure amplitude with circulation ratio. Solid symbols: $\alpha=0.2, C / R=4$; open symbols: $\alpha=0.4, C / R=4 . \bigcirc: p_{1} ; \square$ : $p_{2} ; \triangle: p_{3} . \longrightarrow: p \propto(\gamma-1)^{2} ;-\cdot-: p \propto \gamma^{3} ;--: p \propto \gamma^{4}$.

the generation of sound. The relationship between the corresponding acoustic terms is basically the same as that observed for $\gamma=8$ and is not shown here. A further decrease in the circulation ratio $\gamma$ to 2 results in more serious deformation of the strong ring core [Fig. 16(b)]. However, the time variation patterns of the far-field pressure fluctuations are not significantly affected and is not presented.

A $98 \%$ drop in the amplitude of the radiated sound is observed when the circulation ratio $\gamma$ is changed from 8 to 2 , showing that $\gamma$ has a substantial impact on the sound radiation. Figure 17 shows the variations of the sound amplitude at the pass-through instant $p_{1}$, the first maximum pressure amplitude after the pass-through instant $p_{2}$, and the pressure amplitude of the wavy oscillations $p_{3}$ when the vortex ring cores are propagating away from each other with circulation ratio $\gamma$. It is noted that $p_{3}$ is the pressure amplitude due solely to the axial jerking motion of the strong ring. At $\alpha=0.4, p_{2}$ is larger than $p_{1}$ at a circulation ratio $\gamma=10$. Further evaluation of the data suggests there exist simple relationships among $p_{1}, p_{2}$, and $\gamma$ as $p_{1} \propto(\gamma-1)^{2.04}$ and $p_{2} \propto \gamma^{3.1}$ (Fig. 17). The correlation coefficients are better than 0.99 . Thus the sound radiated at the pass-through instant is nearly proportional to the square of the initial difference in the vortex ring circulation magnitudes. As the initial vortex ring velocity is proportional to its circulation when $\alpha$ and $\lambda$ are fixed, $\gamma$ is actually the ratio of initial vortex ring velocities. The factor $(\gamma-1)$ represents the difference in the initial speeds of the vortex rings relative to that of the weak ring. As there is no pass-though process during head-on collision of vortex ring of equal strength $(\gamma=1), p_{1} \rightarrow 0$ as $\gamma \longrightarrow 1$, agreeing with the relationship of $p_{1}$ and $\gamma$ mentioned above. $p_{2}$, on the other hand, depends mainly on the third power of the initial velocity ratio of the vortex rings. The solid symbols in Fig. 17 indicate that similar relationships among $p_{1}$, $p_{2}$, and $\gamma$ exist at $\alpha=0.2$, when the deformation of vortex ring cores is not significant. Therefore, it can further be concluded that the sound intensity generated by collision at or near the pass-through instant is proportional to the fourth power of the difference in the speeds of the vortex rings. Just after the pass-through instant, the sound intensity is proportional to the sixth power of ring velocity ratio. The amplitude of the wavy oscillations in the far-field pressure fluctuations, where the vortex rings are moving away from each other, depends on the fourth power of the velocity ratio (Fig. 17), suggesting that the sound intensity is proportional to the eighth power of vortex ring velocity ratio at their increased flight time.

For fixed core size $\alpha$ and circulation ratio $\gamma$, the increase in the vorticity constant $\lambda$ increases the total circulation of the system but does not change the pattern of the vortex ring interaction though the amplitude of the far-field pressure fluctuations is different. A doubling of $\lambda$ results in a doubling of both the vortex circulation and velocity. It also results in a $15 \times$ increase in the sound-pressure fluctuation amplitude (not shown here). This is consistent with the eight power law of sound intensity radiation by turbulence flow of Lighthill. ${ }^{21}$ However, the change in the amplitude of the far-field pressure fluctuations, due to the increase in $\alpha$ from 0.2 to 0.4 with fixed $\gamma$ and $\lambda$, is not related to the fourth power of the vortex ring velocity. Instead, the power is close to 3.5 for $p_{1}$, 4.2 for $p_{2}$, and 4.8 for $p_{3}$. As the major difference between the interaction at $\alpha=0.2$ and 0.4 is the extent of core deformation, the above findings suggest that the sound intensity radiated also depends on the deformation of the vortex ring cores and thus the type of interaction involved. However, the patterns of far-field pressure fluctuation time variations are similar to those shown in Figs. 8 and 14.

\section{CONCLUSIONS}

The vorticity dynamics and the acoustic radiation due to the collision of two vortex rings are studied numerically using the method of contour dynamics. Instead of presenting only the total sound radiated by the interaction as other researchers did, the individual contributions from various dynamic parameters of the vortex rings such as velocity, acceleration, and jerk in the radiated sound are also investigated and presented.

In the collision of equal strength vortex rings, it is found that the axial jerks of the rings are relatively more important than their accelerations and velocities in the generation of sound, especially when the vortex rings are thin and before their cores are very close to each other. The importance of the axial jerk decreases with increasing vortex core size.

When the strengths of the vortex rings are not equal, the deformations of vortex ring cores depend on the effective core radii and the circulation ratio. For a small core radius the deformations experienced by the vortex ring cores are negligible. The sound amplitude reaches its maximum value when the vortex rings are coplanar at the pass-through instant and this maximum sound pressure is highly related to the high radial acceleration of the weak ring. In addition, wavy oscillations appear on the far-field sound-pressure time traces as the two vortex rings move away from each other after the pass-through instant. These oscillations are associated with the axial jerk of the strong ring. The amplitude of such oscillations increases with the fourth power of the circulation ratio of the vortex rings. 
The increase in the effective core size results in severe deformation of the vortex ring cores, especially that of the weak ring, after the pass-through instant. After this instant, the vorticity centroid of the weak ring then moves very slowly such that the sound radiation depends mainly on the motions of the strong ring. The mutual induction strength between the vortex rings decreases as they separate from each other such that the change in the impulse of the strong ring becomes small some time after the pass-through instant. The axial jerk of the strong ring then becomes important in the sound generation.

The present results also suggest that the maximum amplitude of sound intensity generated by collision of vortex rings of different strengths at, and close to, the pass-through instant is nearly proportional to the sixth power of the circulation ratio. It is also nearly proportional to the seventh power of the vortex ring velocity with a change in core size but to the eighth power of the velocity with an increase in circulation without any change in the core size.

\section{ACKNOWLEDGMENTS}

The work was partly supported by a donation from Dr. Haking Wong and the Croucher Foundation.

\section{APPENDIX: THE METHOD OF CONTOUR DYNAMICS}

As discussed by Pullin, ${ }^{16}$ contour dynamics refer to the numerical solution of initial value problems for piecewise constant vorticity distributions by the Lagrangian method of calculating the evolution of the vorticity jumps. The following paragraphs, which outline the method, are based on the work of Pozrikidis. ${ }^{17}$

In an inviscid, axisymmetric nonswirling flow and for cylindrical polar coordinates $(z, \sigma, \theta)$, the velocity at a point in the flow $(u, v, 0)$ can be expressed in terms of the Stokes stream function $\psi$ as

$$
\mathbf{u}=(u, v, 0)=\boldsymbol{\nabla} \times(\psi / \sigma \hat{\mathbf{k}})=\frac{1}{\sigma}\left(\frac{\partial \psi}{\partial \sigma},-\frac{\partial \psi}{\partial z}, 0\right) .
$$

It can be shown that if there is a vortex ring of radius $\sigma_{0}$ at an axial distance $z_{0}$ from the origin, the stream function at an arbitrary point in the flow field is given by the equation

$$
\psi(z, \sigma, \theta)=\int G \omega d \sigma d z
$$

where

$$
G=\frac{1}{2 \pi}\left(\sigma_{0} \sigma\right)^{1 / 2}[(2 / k-k) K(k)-2 E(k) / k],
$$

with

$$
k^{2}=4 \sigma_{0} \sigma /\left[\left(z-z_{0}\right)^{2}+\left(\sigma+\sigma_{0}\right)^{2}\right] .
$$

$K$ and $E$ are, respectively, the complete elliptic integrals of the first and second kind. $\psi$ is actually independent of azimuthal angle $\theta$, and $G$ is the stream function associated with a circular vortex ring of unit strength. If $\psi$ is constant across the cross section of the vortex ring and the ring core is small, Eqs. (A1)-(A4) reduce to the Biot-Savart induction formula employed by Kambe and Minota. ${ }^{13}$
Substituting Eq. (A2) into Eq. (A1), one obtains

$$
(u, v, 0)=\int \nabla \times\left(\frac{\psi}{\sigma} \hat{\mathbf{k}}\right) d \sigma d z .
$$

As $\omega$ is independent of $z$,

$$
v=-\frac{1}{\sigma} \int \frac{\partial}{\partial z_{0}}(G \omega) d \sigma d z
$$

Similarly, one observes that $\mathbf{u}=\boldsymbol{\nabla} \phi$, where $\phi$ denotes the velocity potential at the point of interest. Then, it can be shown that

$$
\phi=-\int \frac{\Omega \omega}{4 \pi} d \sigma d z
$$

where $\Omega$ is the solid angle subtented by the ring from the point of interest. One arrives at the formula

$$
u=-\int \frac{\partial}{\partial z_{0}}\left(\frac{\Omega \omega}{4 \pi}\right) d \sigma d z
$$

Equation (1) can be obtained by applying Stoke's theorem to Eqs. (A6) and (A8).

${ }^{1}$ A. Powell, "Theory of Vortex Sound," J. Acoust. Soc. Am. 36, 177-195 (1964).

${ }^{2}$ P. O. A. L. Davies, "Structure of Turbulence," J. Sound Vib. 28, 513-526 (1973).

${ }^{3}$ A. K. M. F. Hussain, "Coherent Structures-Reality and Myth," Phys. Fluids 26, 2816-2850 (1983).

${ }^{4}$ J. E. Bridges and A. K. M. F. Hussain, "Roles of Initial Conditions and Vortex Pairing in Jet Noise," J. Sound Vib. 117, 289-311 (1987).

${ }^{5}$ J. Laufer, "On the Mechanism of Noise Generation by Turbulence," in Omaggio a Carlo Ferrari (Libreria Editrice Universitaria Levrotto and Bella, Torino, 1974), pp. 451-464.

${ }^{6}$ S. K. Tang and N. W. M. Ko, "A Study on the Noise Generation Mechanism in a Circular Jet," ASME J. Fluids Eng. 115, 425-435 (1993).

${ }^{7}$ S. K. Tang and N. W. M. Ko, "Coherent Structure Interaction in Unexcited Coaxial Jet of Mean Velocity Ratio 0.3," Exp. Fluids 17, 147-157 (1994).

${ }^{8}$ H. K. Tanna, "Coannular Jets-Are They Really Quiet and Why?," J. Sound Vib. 72, 97-118 (1980).

${ }^{9}$ S. K. Tang and N. W. M. Ko, "Vortex Interaction in Unexcited Coaxial Jet of Mean Velocity Ratio of 0.3," Proceedings of the 5th Asian Congress on Fluid Mechanics, Korean Institute of Science and Technology, Taejon, Korea, 1992, pp. 579-582.

${ }^{10}$ K. Shariff and A. Leonard, "Vortex Rings," Annu. Rev. Fluid Mech. 24, 235-279 (1992).

${ }^{11}$ Y. Oshima, "Head-on Collision of Two Vortex Rings," J. Phys. Soc. Jpn. 44, 328-331 (1978).

${ }^{12}$ N. Riley, "On the Behaviour of Pairs of Vortex Rings," Q. J. Mech. Appl. Math. 46, 521-539 (1993).

${ }^{13}$ T. Kambe and T. Minota, "Acoustic Wave Radiated by Head-on Collision of Two Vortex Rings,” Proc. R. Soc. London Ser. A 386, 277-308 (1983).

${ }^{14}$ K. Shariff, A. Leonard, N. J. Zabusky, and J. H. Ferziger, "Acoustics and Dynamics of Coaxial Interacting Vortex Rings," Fluid Dyn. Res. 3, 337343 (1988).

${ }^{15}$ W. Möhring, "On the Vortex Sound at Low Mach Number," J. Fluid Mech. 85, 685-691 (1978).

${ }^{16}$ D. I. Pullin, "Contour Dynamics Methods," Annu. Rev. Fluid Mech. 24, 89-115 (1992).

${ }^{17}$ C. Pozrikidis, "The Nonlinear Instability of Hill's Vortex," J. Fluid Mech. 168, 337-367 (1986)

${ }^{18}$ J. Norburg, "A Family of Steady Vortex Rings," J. Fluid Mech. 57, 417431 (1973).

${ }^{19}$ H. Lamb, Hydrodynamics (Dover, New York, 1953).

${ }^{20}$ W. M. Hicks, "On the Mutual Threading of Vortex Rings," Proc. R. Soc. London Ser. A 102, 111-131 (1922).

${ }^{21}$ M. J. Lighthill, "On Sound Generated Aerodynamically II. Turbulence as a Source of Sound," Proc. R. Soc. London Ser. A 222, 1-32 (1954). 